\title{
INVESTIGATIONS OF HIGH TEMPERATURE PHASE TRANSITIONS IN $\left(\mathrm{NH}_{3} \mathrm{C}_{2} \mathrm{H}_{5}\right)_{2} \mathrm{CuCl}_{4}$ CRYSTALS
}

\author{
V. Kapustianik, Yu. Korchak, S. Sveleba \\ Laboratory of Ferroics Physics, Physical Dept., Lviv Franko University \\ Kyryla i Mefodiya st. 8, 290005 Lviv-5, Ukraine \\ R. TCHUKVINSKYI \\ Institute of Experimental Physics, University of Wrocław \\ Pl. M. Borna 9, 50-204 Wrocław, Poland
}

AND I. GIRNYK

Institute of Physical Optics, Dragomanova st. 23, 290005 Lviv-5, Ukraine

(Received December 9, 1998)

On the basis of dilatometric, optical, and dielectric investigations of $\left(\mathrm{NH}_{3} \mathrm{C}_{2} \mathrm{H}_{5}\right)_{2} \mathrm{CuCl}_{4}$ crystals the existence of phase transitions at $T_{1}=364$ and $T_{2}=356 \mathrm{~K}$ was confirmed. Anomalous behaviour of the thermal expansion coefficient and optical birefringence around $T_{3}=330 \mathrm{~K}$ was related to earlier unknown phase transition. Besides, it was shown that these crystals are characterised by considerable protonic conductivity, the nature of which is fairly well explained in the framework of Grotthus mechanism of proton transport.

PACS numbers: 64.70.-p

\section{Introduction}

The crystal of ethylammonium tetrachlorocuprate $\left(\mathrm{NH}_{3} \mathrm{C}_{2} \mathrm{H}_{5}\right)_{2} \mathrm{CuCl}_{4}$ (abbreviately $\mathrm{EA}-\mathrm{CuCl}_{4}$ ). should be considered as two-dimensional materials which exhibit strong structural anisotropy. They consist of ionic layers of $\mathrm{CuCl}_{6}$ octahedra sandwiched between the two sheets of $\left(\mathrm{C}_{2} \mathrm{H}_{5} \mathrm{NH}_{3}\right)^{+}$complexes $[1,2]$. The $\mathrm{NH}_{3}$ polar head of the alkylammonium ion points toward the layer of $\mathrm{CuCl}_{6}$ and forms hydrogen bonds with $\mathrm{Cl}^{-}$anions in $\mathrm{CuCl}_{6}$. The alkyl residue points away from the layer and thus forms neutral strata. They are piled up and bound by the Van der Waals interaction between the alkyl heads. Thus the $\mathrm{EA}-\mathrm{MeCl}_{4}$-type 
crystals become appropriate substances for investigations of the two-dimensional electronic system. These compounds show a number of phase transitions due to the arrangement of the alkylammonium chain. The hydrogen bonds change drastically with the crystal temperature [1].

According to [2] $\mathrm{EA}-\mathrm{CuCl}_{4}$ crystals possess the following sequence of phase transitions (PTs):

$$
\begin{aligned}
& \text { IV III II I } \\
& \text { tryc. } \stackrel{T_{4}=232}{\longleftrightarrow} P_{B c a} \stackrel{T_{2}^{\mathrm{h}}=356 \mathrm{~K}}{\underset{T_{2}^{\mathrm{c}} \stackrel{\rightleftarrows}{\rightleftarrows}}{\rightleftarrows}} \quad P 2_{1} / c \stackrel{T_{1}=364 \mathrm{~K}}{\longleftrightarrow} \quad B b c m .
\end{aligned}
$$

Following optical and spectral investigations confirmed availability of the phase transitions in $\mathrm{EA}-\mathrm{CuCl}_{4}$ at $T_{1}=364 \mathrm{~K}, T_{2}^{\mathrm{h}}=356 \mathrm{~K}, T_{4}=232 \mathrm{~K}[3,4]$, although it was supposed that anomalous behaviour of investigated spectral properties observed both at heating and cooling around the temperature of $T=330 \mathrm{~K}$ should be related to earlier unknown phase transition [4]. Such an ambiguity demands further study of the high temperature phase transitions using more precise methods. This paper, particularly, describes the dilatometric, dielectric and birefringent properties of $\mathrm{EA}-\mathrm{CuCl}_{4}$ crystals in the noted temperature region.

\section{Experimental}

Single crystals of $\left(\mathrm{NH}_{3} \mathrm{C}_{2} \mathrm{H}_{5}\right)_{2} \mathrm{CuCl}_{4}$ were grown at $40^{\circ} \mathrm{C}$ by slow evaporation of a saturated aqueous solution of $\mathrm{CuCl}_{2}$ and $\left(\mathrm{NH}_{3} \mathrm{C}_{2} \mathrm{H}_{5}\right) \mathrm{Cl}$ salts taken in the stoichiometric ratio. The crystalline platelets of different thickness, freshly cleaved along the crystalline layers, were used in all experiments. To avoid the influence of absorption water the samples were previously annealed at the temperatures higher than $370 \mathrm{~K}$.

Thermal expansion was investigated with employment of the quartz dilatometer with a capacity sensor. The temperature increments of the optical birefringence were measured by Senarmont's method. In the dielectric investigations conducting silver-paste electrodes were applied on the previously polished samples. The dielectric parameters were measured with an accuracy of nearly $1 \%$ at different frequencies of the measuring electric field $(100 \mathrm{~Hz}$ to $1 \mathrm{MHz})$ using the Hewlett-Packard 4275 A multifrequency LCR-meter. The applied ac electric field did not exceed $10 \mathrm{~V} / \mathrm{cm}$.

\section{Results and discussion}

Important information about apparent phase transitions in $\mathrm{EA}-\mathrm{CuCl}_{4}$ would be obtained from the data of dilatometry. The temperature dependence of the thermal expansion coefficient $\alpha$ obtained in a heating run is depicted in Fig. 1. This parameter manifests clear peak-like anomalies at the temperatures $T_{1}=364 \mathrm{~K}$, $T_{2}=356 \mathrm{~K}$ and $T_{3}=330 \mathrm{~K}$ reflecting a considerable change of the thermal dilatation behaviour in the different crystalline phases. Existence of the phase transitions at mentioned temperature points is also confirmed by the temperature dependence of optical birefringence (Fig. 2). Indeed, the jumps of $\delta(\Delta n)_{\mathrm{c}}$ observed at $T_{1}$ and $T_{2}$ should be related to the first-order PTs, whereas a slight but noticeable change 


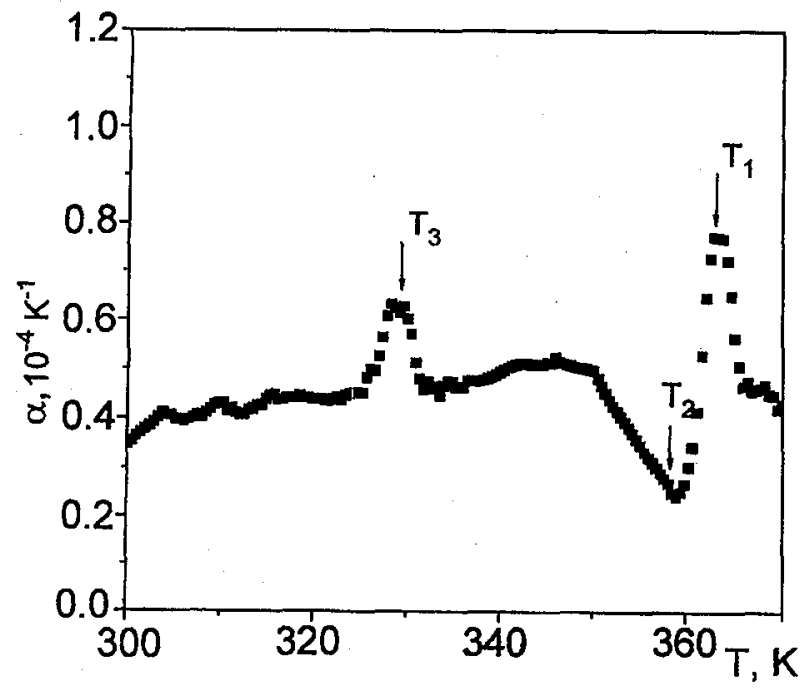

Fig. 1. Temperature dependence of the thermal expansion coefficient measured along the [001] direction of $\mathrm{EA}-\mathrm{CuCl}_{4}$ single crystal.

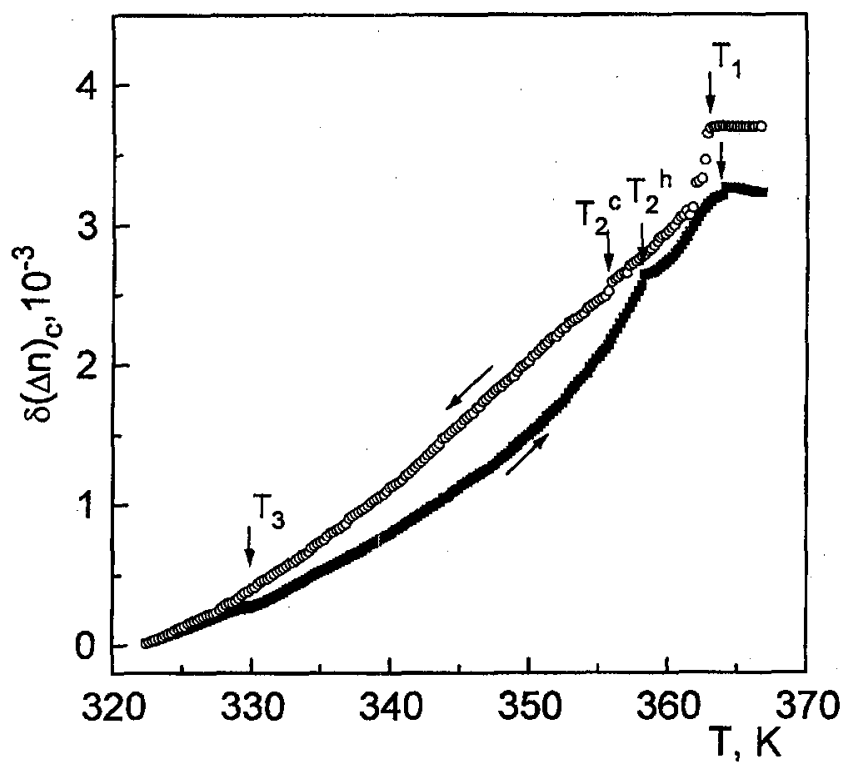

Fig. 2. Temperature dependences of the optical birefringence increment obtained in cooling and heating runs.

of slope at $T_{3}$ is rather characteristic of the second-order PT. Discontinuous nature of both high temperature transitions is confirmed by their temperature hysteresis $\left(\Delta T_{1}=1.5 \mathrm{~K}\right.$ and $\left.\Delta T_{2}=2.5 \mathrm{~K}\right)$. Nevertheless, the obtained value of $\Delta T_{2}$ is much lower than those presented in [2]. Such a discrepancy would be connected with a 
different sample quality and preceding thermal history. In particular, it has been found [2] that $\Delta T_{2}$ varies between 10 and $23 \mathrm{~K}$ in strained crystals. In the same time, one can conclude that behaviour of thermal expansion and birefringence testifies to the existence of an additional phase transition at $T_{3}=330 \mathrm{~K}$. Such a conclusion correlates with the information about the two-stage transformation of domain structure taking place according to [2] in a heating run both in vicinity of the temperatures $T_{2}^{\mathrm{c}}$ and $T_{2}^{\mathrm{h}}$ (see the phase sequence presented above). Therefore, it is convenient to suppose that the first of these temperatures obtained in [2] in our case corresponds to the PT at $T_{3}$.

Strongly marked two-dimensional structure of $\mathrm{EA}-\mathrm{CuCl}_{4}$ and presence of the organic molecules with a dipole moment would assume interesting dhelectric properties. As one can see from Figs. 3 and 4, the dielectric parameters $\varepsilon^{\prime}$ and $\operatorname{tg} \delta$ do not show any pronounced anomalies at $T_{3}$. Therefore, if there is a $\mathrm{PT}$ in this point, its nature is not connected with ferroelectricity or any drastic change of the dielectric relaxation. At the same time, one can note that marked dielectric dispersion in the investigated frequency window is developed just above $T_{3}=$ $330 \mathrm{~K}$. On the other hand, PT at $T_{2}$ manifests itself by the sharp anomalies both of dielectric permittivity and losses. Due to observed dielectric dispersion it seems that these anomalies are a little shifted in respect of the temperature depending on the frequency of measuring electric field. It is necessary to note that dispersion of the dielectric parameters in the frequency region of $1 \mathrm{kHz} \div 1 \mathrm{MHz}$ should be considered as a characteristic feature of compounds with alkylammonium cation and usually is related to the relaxation process connected with reorientation of the organic molecules [5-8]. Indeed, the ethylammonium groups are disordered

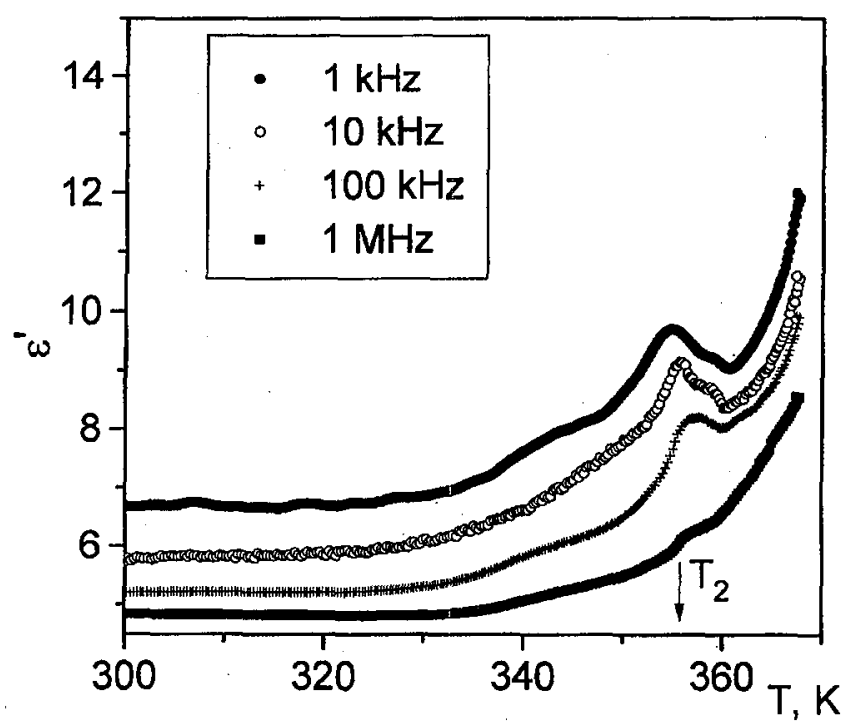

Fig. 3. Temperature dependences of the dielectric permittivity measured in a heating run on the c-cut of $\mathrm{EA}^{-\mathrm{CuCl}} \mathrm{Cu}_{4}$ at different frequencies of measuring electric field. 


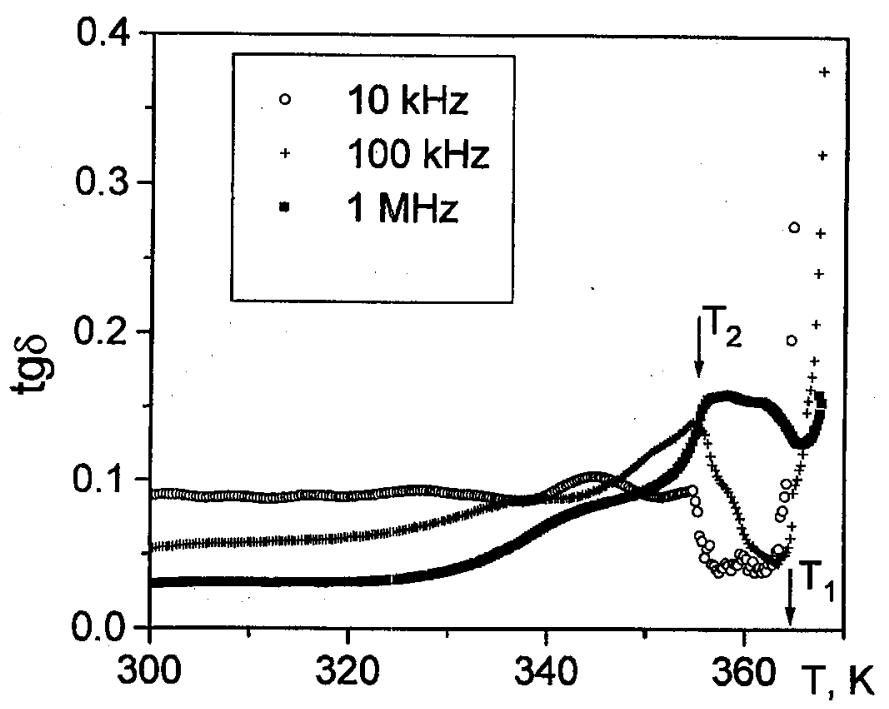

Fig. 4. Temperature dependences of the dielectric loss tangent measured in a heating run on the $c$-cut of $\mathrm{EA}-\mathrm{CuCl}_{4}$ at different frequencies of measuring electric field.

in the high temperature phase of $\mathrm{EA}^{-\mathrm{CuCl}_{4}}$ and their chains flip between the four equivalent states of the $\mathrm{NH}_{3}$ group in the chlorine cavity. With decreasing temperature the motion "freezes in" stepwise at phase transitions. Therefore, the organic complexes become ordered by such a way that ethylammonium groups above and below the copper chlorine plane are transformed into each other by a centre of inversion [2]. Described pattern fairly well correlates with stepwise growing of dielectric dispersion at heating.

With further heating high dc conductivity arising in vicinity of $T_{1}$ makes impossible correct measurements of the dielectric permittivity. In the same time, study of the low frequency conductivity presents special interest since the investigated crystals containing the hydrogen bonds in their structure would manifest considerable protonic conductivity $[9,10]$.

The conductivity was measured at frequencies not higher than $1 \mathrm{kHz}$ to avoid the influence of dielectric relaxation processes occurring at higher frequencies. As one can see from Fig. 5, the conductivity reaches maximum values in the phase II lying in the framework of $T_{1} \div T_{2}$ and grows exponentially with temperature. The temperature dependence of $\sigma$ manifests clear anomalies at the temperatures limiting mentioned phase (with accounting of the temperature hysteresis). One can note that observed comparatively high conductivity by their value even exceeds the conductivity in known protonic conductor such as $\left(\mathrm{NH}_{2}\left(\mathrm{CH}_{3}\right)_{2}\right)_{2} \mathrm{CoCl}_{4}$ [9].

It is interesting to note that electric conductivity within the considered temperature region varies according to Arrhenius behaviour (Fig. 5, insert)

$$
\sigma=\sigma_{0} \exp \left(-\frac{E_{\mathrm{a}}}{k T}\right),
$$

where $\sigma_{0}$ is a pre-exponential factor and $E_{\mathrm{a}}$ is the activation energy of conduc- 


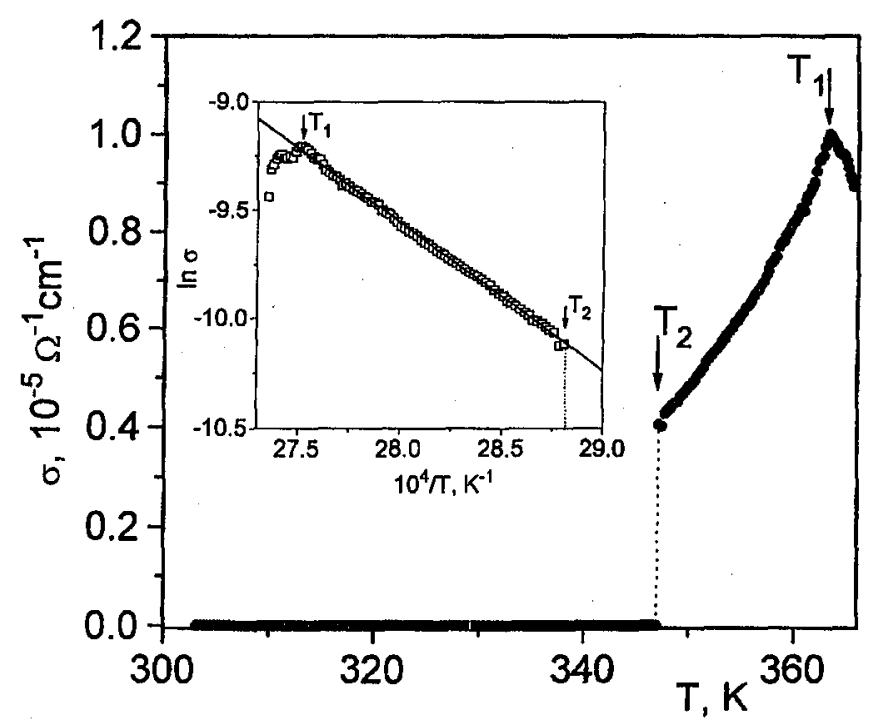

Fig. 5. Temperature dependence of electric conductivity along [001] direction measured in a cooling run at frequency of electric field $f=1 \mathrm{kHz}$. Insert - inverse temperature dependence of $\ln \sigma . \sigma$ in $\Omega^{-1} \mathrm{~cm}^{-1}$.

tivity. The latter is equal to $E_{\mathrm{a}}=0.585 \pm 0.005 \mathrm{eV}$. Taking into account that dependence $\ln \sigma=f(1 / T)$ is rectilinear within the whole phase II, one can suppose that pre-exponential factor practically does not depend on temperature.

On the basis of available structural [2] data one can suppose that the protonic conductivity is connected with the so-called "Grotthus" mechanism which includes correlated jumps of the bare proton between host molecules and correlated reorientation of the latter [10]. In this case the ethylammonium cations would be treated as host molecules. In considered phase they can occupy two possible positions determined by directions of the $\mathrm{N}-\mathrm{H}$.. . Cl bonds. One can suggest that co-operative reorientation of the organic groups proceeds in the copper-chlorine plane whereas the translocation of protons between the neighbouring sheets of organic molecules is observed in the perpendicular direction. In this case to permit proton transport the activation energy of conductivity has to be larger than both activation energy of host molecule reorientation and the energy barrier between two different H-bonds [2]. Unfortunately, it is difficult to investigate in detail the dielectric relaxation process because of very narrow region of existence of phase II. Nevertheless, one can note that determined value of $E_{\mathrm{a}}$ is higher than in related compounds with alkylammonium-cation $\left(E_{\mathrm{a}}=0.340 \mathrm{eV}\right.$ for $\left(\mathrm{NH}_{2}\left(\mathrm{CH}_{3}\right)_{2}\right)_{2} \mathrm{CoCl}_{4}$ [9] and $E_{\mathrm{a}}=0.355 \mathrm{eV}$ for $\mathrm{NH}_{2}\left(\mathrm{CH}_{3}\right)_{2} \mathrm{MnCl}_{3} \cdot 2 \mathrm{H}_{2} \mathrm{O}$ [8]), first of all, due to larger energy barrier between two different $\mathrm{H}$-bonds [2].

\section{Conclusion}

Optical, dilatometric and dielectric study confirmed the existence of first-order phase transitions in $\mathrm{EA}-\mathrm{CuCl}_{4}$ crystals at temperatures: $T_{1}=364 \mathrm{~K}$ and 
$T_{2}=356 \mathrm{~K}$ (in a heating run). Besides, the anomalous behaviour of investigated parameters around $T_{3}=330 \mathrm{~K}$ would testify to occurrence of one more phase transition at this temperature point. Unfortunately, available structural data [2] are not sufficient to confirm the existence of this transition because of its continuous character and too large step of temperature change at the measurements. It has been also found that $\mathrm{EA}-\mathrm{CuCl}_{4}$ crystals in the phase II are characterised by pronounced protonic conductivity, which is realised through the Grotthus mechanism. Nevertheless, further microscopic investigations are necessary in order to clarify the nature of proton transport and the mechanism of probable structural phase transition at $T_{3}$.

This work was partially supported by the International Science Foundation in the framework of ISEP program.

\section{References}

[1] T. Yoshinari, T. Nanba, S. Shimanuki, M. Fujisawa, T. Matsuyama, M. Ikezawa, K. Aoyagi, J. Phys. Soc. Jpn. 58, 2276 (1989).

[2] I.R. Jahn, K. Knorr, J. Ihringer, J. Phys., Condens. Matter 1, 6005 (1989).

[3] V. Kapustianik, Yu. Korchak, I. Polovinko, R. Tchukvinskyi, Z. Czapla, S. Dacko, Phys. Status Solidi B 207, 95 (1998).

[4] V.B. Kapustianik, Yu.M. Korchak, I.I. Polovinko, Ukrainskyi Fizychnyi Zhurnal, 1998 , to be published.

[5] K. Sano, S. Kashida, J. Phys. C., Solid State Phys. 20, 2585 (1987).

[6] V.B. Kapustianik, H. Kabelka, H. Warhanek, A. Fuith, Phys. Status Solidi A 155, 95 (1996).

[7] V.B. Kapustianik, Yu. Korchak, V. Mokryi, V. Dzhala, I. Polovinko, S. Dacko, Z. Czapla, Ukrainskyi Fizychnyi Zhurnal 42, 1058 (1997).

[8] V. Kapustianik, Phys. Status Solidi A 168, 109 (1998).

[9] V.B. Kapustianik, S.A. Sveleba, R. Tchukvinskyi, Yu. Korchak, V. Mokryi, I.I. Polovinko, Z. Trybula, Phys. Status Solidi A 151, 481 (1995).

[10] Proton Conductors, Solid, Membranes and Gels - Materials and Devices, Ed. P. Colomban, Cambridge University Press, Cambridge 1992. 